\title{
Comunicación
}

\section{Uso de la oxitetraciclina en el tratamiento de la ehrlichiosis canina: estudio retrospectivo de 15 casos en Camagüey, Cuba}

\author{
USE OF OXYTETRACYCLINE IN THE TREATMENT OF CANINE EHRLICHIOSIS: A RETROSPECTIVE \\ STUdy OF 15 Cases in Camagüey, Cuba \\ Iván Peña G. ${ }^{1,3}$, Florangel Vidal F. ${ }^{1}$, Arnaldo del Toro R. ${ }^{2}$, Aliesky Hernández R. ${ }^{1}$
}

\section{Resumen}

Se hizo un estudio retrospectivo de 15 pacientes caninos de los barrios del municipio Camagüey, Cuba, en el periodo 2016-2017, con signos clínicos compatibles con hemoparasitosis y con diagnóstico de laboratorio mediante frotis sanguíneo y coloración Giemsa de ehrlichiosis canina. Se propuso una terapia alternativa con reconstituyentes antianémicos, hidratación con Lactato Ringer y oxitetraciclina para el tratamiento de la ehrlichiosis canina.

Palabras clave: canino; hemoparásito; Rhipicephalus sanguineus; ehrlichiosis canina

\section{ABstract}

A retrospective study of 15 canine patients from the neighborhoods of Camagüey, Cuba in the period 2016-2017, with clinical signs compatible with hemoparasitosis and laboratory diagnosis by blood smear and Giemsa coloration of canine ehrlichiosis. An alternative therapy with antianemic reconstitutes, hydration with Lactate Ringer and oxytetracycline was proposed for the treatment of canine ehrlichiosis.

Key words: canine; hemoparasito; Rhipicephalus sanguineus; canine ehrlichiosis

\footnotetext{
${ }^{1}$ Universidad de Camagüey Ignacio Agramonte Loynaz, Cuba

${ }^{2}$ Facultad de Medicina Veterinaria, Universidad Técnica de Manabí, Ecuador

${ }^{3}$ E-mail: ivan.pena@reduc.edu.cu
} 
INTRODUCCIÓN

Entre las enfermedades que afectan a las mascotas se encuentran las causadas por hemoparásitos, las que representan gran importancia veterinaria, ya que en casos agudos pueden conducir a la muerte del animal. Además, algunas son de carácter zoonótico, cuyo riesgo de infección se agrava por la intensa relación entre las mascotas y los dueños (Andrade et al., 2016).

La ehrlichiosis es producida por un organismo perteneciente al subgrupo $\alpha$ Proteobacteria, orden Rickettsiales, familia Anaplasmataceae, género Ehrlichia. Se caracteriza por ser una bacteria intracelular obligada pequeña (0.4-1.5 $\mu \mathrm{m})$, Gram-negativa, generalmente redonda, aunque algunas veces altamente pleomórfica, que se replica dentro de una vacuola derivada de la membrana de la célula eucariota del hospedero vertebrado o invertebrado (Dumler et al., 2001; Ismail et al., 2010). La enfermedad se transmite en los perros a través de garrapatas como Rhipicephalus sanguineus, y el agente patógeno se disemina vía sanguínea o linfática dentro de las células mononucleares infectadas, llegando a otros sistemas orgánicos. La mayoría de casos se presenta durante los meses de primavera y verano, cuando la población de garrapatas es más activa (Huerto y Dámaso, 2015).

Las garrapatas son ectoparásitos hematófagos obligados (Bowman, 2009); Cortés y Forero, 2013) de prácticamente todos los vertebrados terrestres, principalmente mamíferos y aunque han sido consideradas parásitos cosmopolitas, numerosas especies están restringidas a regiones específicas (Needham y Teel, 1991; Anderson, 2002; Guglielmone et al., 2004; Hernández, 2005; Pereira et al., 2008; Estrada-Peña, 2008; Cortés y Forero, 2013). Se reconocen alrededor de 907 especies de garrapatas distribuidas en todo el mundo, pero especialmente concentradas en las regiones tropicales y subtropicales (Davey et al., 1997; Wall y
Shearer 2001; Horak et al., 2002: Barker y Murrel 2008; Cortés y Forero 2013).

El presente estudio propone una alternativa de terapia contra hemoparásitos usando oxitetraciclina en el tratamiento de la ehrlichiosis canina.

\section{Materiales y MéTodos}

\section{Generalidades}

La provincia Camagüey presenta un clima de llanuras, principalmente interiores, con alta humedad y evaporación estacional y elevada temperatura ambiental. Temperatura mínima promedio de $22.7^{\circ} \mathrm{C}$ y máxima promedio de $28.9{ }^{\circ} \mathrm{C}$, con precipitación pluvial anual de $1200-1400 \mathrm{~mm}$, con el $70-86 \%$ en entre mayo y octubre. La topografía es llana, entre 100 y 200 msnm (Atlas de Camagüey, 1990).

\section{Casos Clínicos}

Se realizó el seguimiento médico a 15 pacientes caninos de diferentes propietarios procedentes de los barrios del municipio Camagüey, Cuba, que fueron atendidos en el periodo 2016-2017, con signos clínicos compatibles con ehrlichiosis canina.

El diagnóstico clínico de la hemoparasitosis se realizó por medio de los hallazgos clínicos compatibles con la enfermedad (Couto y Nelson, 2000; Huerto y Dámaso, 2015), la presencia de garrapatas vectoras que afectan a los caninos como Rhipicephalus sanguineus y Amblyomma cajenense (garrapatas de tres hospedadores), que hacen que las hemoparasitosis estén presentes en el medio (Benavides y Ramírez, 2003).

La investigación fue llevada a cabo bajo las condiciones de Cuba. Debido a limitaciones materiales y obsolescencia tecnológica, el examen de laboratorio para confirmar el 
diagnóstico clínico contempló el método más simple, rápido y económico para detectar la bacteria consistiendo en toma de muestras de sangre, frotis sanguíneo delgado y coloración Giemsa para la visualización del patógeno (Dolz et al., 2013).

En general, los pacientes recibieron tratamiento a base de oxitetraciclina L.A. 20 $\mathrm{mg} / \mathrm{kg}$ (Plumb, 2010), por vía intramuscular cada $72 \mathrm{~h}$, conjuntamente con tratamiento de soporte que incluyó vitaminas del complejo $\mathrm{B}$ con fósforo y aminoácidos de $0.5-2 \mathrm{ml} \mathrm{im}$, cada 7 días; reconstituyente antianémico Ferrodex (Biomont) en dosis de $0.5 \mathrm{ml} / 10 \mathrm{~kg}$ más Hematovit (Biomont) en dosis de $1 \mathrm{ml} / 5$ $\mathrm{kg}$ sc, cada 72 h y fluidoterapia con solución cristaloide de Ringer lactato, suero salino al $0.9 \%$ iv, en los casos que lo ameritaron.

Se cuantificó la asociación entre los factores de riesgo como presencia de $R$. sanguineus y sexo de los pacientes con la ehrlichiosis canina. Para el análisis se usó el programa estadístico SPSS v. 23 con un nivel de significación de $\mathrm{p}<0.05$.

\section{Pacientes}

Caso 1: Koky. Pequinés macho, $7.6 \mathrm{~kg}$ (septiembre-octubre de 2016). Presentó inicialmente claudicación de un miembro, indicándosele antiinflamatorios y analgésicos. El episodio se repitió sumándose rigidez en los miembros posteriores, marcha envarada, dolor en la región lumbosacra y dificultad a la micción. Presencia de garrapatas. Fue tratado con enrofloxacina $10 \% 0.4 \mathrm{ml} \mathrm{c} / 24 \mathrm{~h}$ por infección del sistema genitourinario durante 5-7 días. Los signos clínicos reaparecieron al término del tratamiento, además de epistaxis nasal incontrolable y presencia de pequeñas áreas hemorrágicas en la región ventral. Fue tratado con coagulante, epinefrina, Ringer lactato y oxitetraciclina L.A. $20 \mathrm{mg} / \mathrm{kg} \mathrm{c} / 72$ h, tres dosis. Se recuperó.

Caso 2: Blanquita. Poodle de 5 años, $8 \mathrm{~kg}$ (diciembre de 2016). Presentó inapetencia, debilidad, petequias en la región ventral, fie- bre $\left(40{ }^{\circ} \mathrm{C}\right)$ y adenopatía. Se había tratado anteriormente con penicilina en otra clínica. Se trató con Ringer lactato más oxitetraciclina L.A. $20 \mathrm{mg} / \mathrm{kg} \mathrm{c} / 72 \mathrm{~h}$, tres dosis. Se recuperó.

Caso 3: Brenda, pastora hembra de tres años, $35 \mathrm{~kg}$ (octubre de 2016). Presentó apatía, inapetencia, anemia marcada, fiebre $\left(39.5^{\circ} \mathrm{C}\right)$, ligera secreción sanguinolenta a través de las fosas nasales y deshidratación. Presencia de garrapatas. Fue tratada con oxitetraciclina L.A. $20 \mathrm{mg} / \mathrm{kg} \mathrm{c} / 72 \mathrm{~h}$, tres dosis, más enrofloxacina $5 \mathrm{mg} / \mathrm{kg} \mathrm{c} / 24 \mathrm{~h}$ durante 10 días.

Caso 4: Perra Pastor Belga, $28 \mathrm{~kg}$ (febrero de 2017). Tratada previamente por intoxicación y luego contra Erlichia canis con un antibiótico erróneo en otra clínica veterinaria. Presentó hemorragia en encías, boca y nariz, y lesiones de garrapatas en el cuerpo, edema en extremidades, disnea, pupila dilatada, dolor generalizado, desorientación, debilidad; así como pulso débil e irregular. Se aplicó tratamiento sintomático, pero presentó un shock hipovolémico, paro respiratorio y muerte.

Caso 5: Lara, mestiza hembra, $9 \mathrm{~kg}$ (abril de 2017). Petequias en la región ventral, epistaxis, fiebre $\left(40^{\circ} \mathrm{C}\right)$, diarrea sanguinolenta, con historia de fuerte infestación por garrapatas. Se trata con Ringer lactato $400 \mathrm{ml} \mathrm{iv}$, dexametasona $0.2 \mathrm{ml} \mathrm{sc}$, profen $1 \% 1 \mathrm{ml} \mathrm{sc}$, ácido tranexánico $1 \mathrm{ml}$ im, oxcitetraciclina L.A. $0.9 \mathrm{ml} \mathrm{im} \mathrm{c/72} \mathrm{h} \mathrm{tres}$ dosis. Se recuperó.

Caso 6: Rufo, mestizo macho, $10 \mathrm{~kg}$ (abril de 2017). Presenta petequias en la región ventral, gran cantidad de pulgas y garrapatas. Se trata con oxitetraciclina L.A. $20 \mathrm{mg} /$ $\mathrm{kg}$ im c/72 h tres dosis y Ringer lactato. Mostró una recuperación rápida.

Caso 7: Yony, Pequinés macho, $5.5 \mathrm{~kg}$, (marzo de 2017). Presencia de ectoparásitos y fue tratado contra ecto y edoparásitos. En días posteriores hubo pérdida de peso y pelo hirsuto, ojo derecho con conjuntivitis, secreción serosa nasal, e historia de heces y vómito con 
sangre. Se prescribe dexametasona $0.1 \mathrm{ml}$ $\mathrm{sc} \mathrm{c} / 24 \mathrm{~h}$ por tres días y amoxicilina $0.64 \mathrm{ml}$ $\mathrm{sc} \mathrm{c} / 72 \mathrm{~h}$ por sospecha de gastroenteritis. Tres semanas más tarde presenta dolor indeterminado, sin fiebre y orina escasa y oscura. Se prescribe antibióticos, analgésicos y antiinflamatorio. Dos semanas más tarde rigidez en extremidades posteriores, dolor y marcha envarada. Se sospecha de ehrlichiosis canina y se indica tratamiento con oxitetraciclina $20 \mathrm{mg} / \mathrm{kg} \mathrm{im} \mathrm{c} / 72 \mathrm{~h}$. Se recuperó.

Caso 8: Plutón. Mestizo macho, 2 años, 10 $\mathrm{kg}$ (marzo de 2017). Presentó epistaxis, inapetencia, secreción ocular purulenta. Se aplicó epinefrina diluida (1:100.000) en la cavidad nasal c/ 5 min hasta lograr controlar el sangrado, más ácido tranexánico $30 \mathrm{mg} / \mathrm{kg}$, oxitetraciclina L.A. $20 \mathrm{mg} / \mathrm{kg} \mathrm{c} / 72 \mathrm{~h} 10$ dosis. Se recuperó.

Caso 9: Yogui. Shar Pei macho, $27 \mathrm{~kg}$ (mayo de 2017). Presenta somnolencia, decaimiento, pérdida de peso y mucosas pálidas blanco porcelano. Se detectó aumento de la frecuencia respiratoria, fiebre $\left(40^{\circ} \mathrm{C}\right)$, ruido pulmonar a la auscultación y presencia de garrapatas. Se indica oxcitetraciclina (594 mg), $3 \mathrm{ml}$ im $\mathrm{c} / 72 \mathrm{~h}$, Ferrodex $1.35 \mathrm{ml} \mathrm{c} / 72 \mathrm{~h}$, vitaminas del complejo B c/72 h, Hematovit $5 \mathrm{ml} \mathrm{sc}$ c/72 y mejora alimenticia de alto contenido en hierro. Se recuperó.

Caso 10: Tribilín. Mestizo, $10 \mathrm{~kg}$ (mayo de 2017). Presencia de orina oscura rojiza y disminución del apetito. Temperatura normal, mucosas oculares pálidas, mucosa oral blanco amarillento, disnea, pero sin ruidos respiratorios a la auscultación. Había sido tratado por anemia. Se refiere la presencia de equinos cerca de la casa. Se inicia el tratamiento con reconstituyentes más oxitetraciclina $209 \mathrm{mg}$ c/72 h. Se recuperó.

Caso 11: Yon. Pastor Belga macho, $35 \mathrm{~kg}$ (mayo de 2017). Historia de hemorragia nasal, presencia de caballos y garrapatas en la vecindad. Se observan mucosas orales y ocu- lares pálidas, temperatura rectal normal, disnea y coagulopatía. Se presentaron dos vómitos con contenido hemorrágico. Se aplicó ácido tranexánico $30 \mathrm{mg} / \mathrm{kg}$, hidroterapia con Ringer más reconstituyentes y oxitetraciclina L.A. $20 \mathrm{mg} / \mathrm{kg}$ c/72 h. En los dos días siguientes presentó epistaxis ligera, repitiéndose el tratamiento con ácido tranexánico. Se recuperó.

Caso 12: Sami. Bóxer hembra, $30 \mathrm{~kg}$ (junio de 2017). La dueña refiere presencia de dolor. Se detecta dolores articulares e inflamación de las extremidades. Temperatura de $39.8^{\circ} \mathrm{C}$, estupor e inapetencia, mocosas de coloración normal. Se indicó inicialmente analgésicos y antinflamatorios. Tres días después se agravó el cuadro clínico, presentando mucosas ocular y oral pálidas y gastroenteritis con melena. Se aplicaron los reconstituyentes, oxitetraciclina L.A. 20 $\mathrm{mg} / \mathrm{kg} \mathrm{c} / 72 \mathrm{~h}$ en 7 dosis, fitonadiona vitamina $\mathrm{K} 3 \mathrm{mg} / \mathrm{kg} \mathrm{sc} \mathrm{c} / 24 \mathrm{~h}$, y protector gástrico Omeprazol $1 \mathrm{mg} / \mathrm{kg}$ oral c/24 h. Se recuperó.

Caso 13: Koky, perro del Caso 1 (julio de 2017). La dueña refiere dolor al caminar y sin micción desde la noche anterior. Presenta caída del tren posterior, marcha envarada o con rigidez, dolor en los miembros, y se desplaza con dificultad. Presencia de garrapatas. No hay ruidos respiratorios y mucosas ligeramente pálidas. Los signos son similares al proceso que tuvo en 2016. Se inicia con el protocolo establecido para este tipo de casos, suministrándose la primera dosis de oxitetraciclina y suero salino al $0.9 \%$. Se recuperó.

Caso 14: Shakira, Chow Chow hembra, $15 \mathrm{~kg}$ (agosto de 2017). La dueña refiere sangrado y presencia de garrapatas. Se detecta epistaxis nasal abundante que no coagulaba, disnea, mucosas pálidas, sin fiebre ni ruidos respiratorios a la auscultación. Se aplica la primera dosis de oxitetraciclina y a las $48 \mathrm{y}$ $72 \mathrm{~h}$ las otras dos dosis y fue hidratado con Ringer Lactato. Se recuperó. 
Caso 15: Yanko. Pastor Belga macho, $35 \mathrm{~kg}$ (agosto de 2017). Fue llevado a otra clínica veterinaria y tratado con vitamina $\mathrm{K}$. Al día siguiente repite la epistaxis y presente heces líquidas y hemorrágicas y se vuelve a tratar con vitamina K. Días más tarde, se presenta a consulta con epistaxis, pérdida del apetito e historia de presencia de garrapatas. Se observa disnea, mucosas oral y ocular muy pálidas, deshidratación, aumento de la frecuencia respiratoria y cardiaca, pero sin fiebre ni ruidos respiratorios. Se aplica la primera dosis de oxitetraciclina y se le hidrata con Ringer Lactato, seguido a las 48 y $72 \mathrm{~h}$ de las otras dos dosis del antibiótico. Se recuperó.

\section{Resultados y Discusión}

La aparición de la mayor parte de casos clínicos se enmarcó en los meses de mayor temperatura, lluvias y humedad, lo cual genera un ambiente propicio para el desarrollo y supervivencia del vector (Cortés y Forero 2013; Alemán et al., 2014). En todos los casos tratados fue visible la presencia de garrapatas, siendo $R$. sanguineus la más frecuente. La mayoría de los dueños de equinos que habitan la ciudad y su periferia no los tratan contra ectoparásitos; asimismo, se puede observar la presencia de perros callejeros (Peña et al., 2016), lo cual facilita que las garrapatas se reproduzcan con facilidad; de allí que $R$. sanguineus pueda parasitar a los perros y transmitir Ehrlichia canis (Huerto y Dámaso, 2015).

La ehrlichiosis canina se inicia usualmente con un proceso agudo caracterizado por depresión, anorexia, letargo, pérdida de peso y fiebre, seguido por una etapa subaguda. En una etapa final, la ehrlichiosis se manifiesta con hemorragias, linfadenopatías, esplenomegalia, poliartropatías y signos neurológicos (Ettinger y Feldman, 1997; Huerto y Dámaso, 2015). En este estudio, los principales síntomas encontrados en los casos de ehrlichiosis canina fueron fiebre, depresión, anorexia, pérdida de peso en la fase aguda y anemia. La fase crónica se caracterizó por hemorragia, epistaxis y edema y poliartritis, aunque también se encontró ataxia y paresia en la fase aguda en los casos de mayor gravedad (Woody y Hoskins, 1991; Breitschwerdt, 1997; Beaufils, 1997; Tasayco et al., 2015).

La oxitetraciclina, antibiótico de la clase de las tetraciclinas, actúa como antibiótico bacteriostático e inhibe la síntesis proteica. También tiene actividad contra la mayoría de las micoplasmas, espiroquetas, clamidias y rickettsias (Plumb, 2010). Para el tratamiento de la ehrlichiosis canina se recomienda la administración de tetraciclina u oxitetraciclina y en el caso de infecciones crónicas con evidencia de falla renal, la doxiciclina (Hoskins, 1991; Tasayco et al., 2015).

La mayor parte de los pacientes se encontraban en la fase aguda de la enfermedad, respondiendo satisfactoriamente al tratamiento con oxitetraciclina L.A. $20 \mathrm{mg} / \mathrm{kg}$ según Plumb (2010) por vía intramuscular, dentro de las primeras 24-72 horas posteriores a la primera administración. Se aplicó la medicación con oxitetraciclina a las 24 horas en los casos más graves a criterio del médico veterinario. La rehidratación con Ringer Lactato fue esencial por ser el cristaloide de elección para iniciar la reanimación del enfermo crítico, exceptuando los casos en los cuales se presentó disfunción renal, pues existe una controversia asociada a su uso y posible causante de fallo renal (GarnachoMontero et al., 2015). Tanto en las unidades de cuidados intensivos de hospitales humanos y veterinarios, uno de los disturbios ácido base más común es la alcalosis metabólica hipoclorémica que en humanos y animales monogástricos resulta principalmente del vómito y de la deshidratación por la diferencia de iones fuertes (Martínez y Oliver, 2016).

El análisis de Odds ratio no indicó diferencias significativas por efecto del sexo, en coincidencia con los estudios de Asgarali et al. (2012) y Cartagena et al. (2015), pero se 
encontró que los perros con garrapatas tenían más probabilidad de ser seropositivos que los perros que no las tenían $(\mathrm{p}<0.05)$.

\section{Literatura Citada}

1. Alemán Y, Martínez S, Corona B. 2014. Las garrapatas de interés veterinario en Cuba, y su importancia en las condiciones climáticas cambiantes. REDVET 15(2). [Internet]. Disponible en: http://www.veterinaria.org/revistas/ redvet/n020214/021409.pdf

2. Anderson F. 2002. The natural history of ticks. Med Clin North Am 86: 205218.

3. Andrade G, Muñoz, Y. 2016. Reporte de caso: infestación por Ehrlichia spp en felino mestizo. REDVET 17(11). [Internet]. Disponible en: http:// www.veterinaria.org/revistas/redvet/ n111116/111610.pdf

4. Asgarali Z, Pargass I, Adam J, Mutani A, Ezeokoli C. 2012. Haematological parameters in stray dogs seropositive and seronegative to Ehrlichia canis in North Trinidad. Ticks Tick Borne Dis 3: 207-211. doi: 10.1016/ j.ttbdis.2012.03.006

5. Atlas de Camagüey. 1989. Camagüey, Cuba: Instituto de Geografía de la Academia de Ciencias de Cuba. $112 \mathrm{p}$.

6. Barker C, Murrell A. 2008. Systematics and evolution of ticks with a list of valid genus and species names. In: Bowman AS, Nutall P (eds). Ticks: biology, disease and control. Cambridge: Cambridge University Press. p 1-39.

7. Beaufils J. 1997. Ehrlichiosis: clinical aspects in dogs and cats. Comp Cont Educ Pract 19: 57-61.

8. Benavides J, Ramírez G. 2003. Ehrlichiosis canina. Rev Col Cienc Pec 16:268-274.

9. Bowman, D. 2009. Arthropods. Ch 2. In: Georgi's parasitology for veterinarians. USA: Saunders. 464 p.
10. Breitschwerdt E. 1997. Ehrlichiosis: one or many diseases? Comp Cont Educ Pract 19: 62-63.

11. Cartagena L, Ríos L, Cardona J. 2015. Seroprevalencia de Ehrlichia canis en perros con sospecha de infección por patógenos transmitidos por garrapatas en Medellín, 2012-2014. Rev Med Vet 29: 51-62. doi: 10.19052/ mv.3446

12. Cortés J, Forero E. 2013. Influencia del calentamiento global en la frecuencia y distribución de garrapatas. En: Mem XL Congreso Colombiano de Entomología. Bogotá.

13. Couto G, Nelson R. 2000. Manual de medicina interna de pequeños animales. Madrid: Ed Harcout y Mousby. 894 p.

14. Davey B, Ahrens H, George E. 1997. Comparative effectiveness of Coumaphos treatments applied by different for the control of Boophilus microplus (Acari: Ixodidae). J Agric Urban Entomol 14: 45-54

15. Dolz G, Ábrego L, Romero L, Campos L, Bouza L, Jiménez A. 2013. Ehrlichiosis y anaplasmosis en Costa Rica. Acta Méd Costarric 55: 34-40.

16. Dumler JS, Barbet AF, Bekker CP, Dasch GA, Palmer GH, Ray SC, Rikihisa Y, Rurangirwa FR. 2001. Reorganization of genera in the families Rickettsiaceae and Anaplasmataceae in the order Rickettsiales: unification of some species of Ehrlichia with Anaplasma, Cowdria with Ehrlichia and Ehrlichia with Neorickettsia, descriptions of six new species combinations and designation of Ehrlichia equi and 'HE agent' as subjective synonyms of Ehrlichia phagocytophila. Int J Syst Evol Microbiol 51:2145-2165.

17. Estrada-Peña A. 2008. Climate, niche, ticks, and models: what they are and how we should interpret them. Parasitol Res 103: S87-S95. doi: 10.1007/s00436-0081056-7 
18. Ettinger SJ, Feldman EC. 1997. Enfermedades del perro y del gato. En: Tratado de medicina interna veterinaria. $4^{\circ}$ ed. Buenos Aires, Argentina: InterMedica. $\mathrm{p}$ 470-484.

19. Garnacho-Montero J, FernándezMondéjar E, Ferrer-Roca R, HerreraGutiérrez M, Lorente J, Ruiz-Santana $S$, Artigas A. 2015. Cristaloides y coloides en la reanimación del paciente crítico. Med Intensiva 39: 389-390. doi: 10.1016/j.medin.2015.03.003

20. Guglielmone AA, Bechara GH, Szabó MP, Barros DM, Faccini JL, Labruna MB, De La Vega R, et al. 2004. Garrapatas de importancia médica y veterinaria: América Latina y El Caribe. The Netherlands: International Consortium on Ticks and Tick-borne Diseases (ICTTD-2). $44 \mathrm{p}$.

21. Hernández F. 2005. El manejo integrado en el control de garrapatas. En: Manual de ganadería doble propósito. Maracaibo, Venezuela: Universidad del Zulia. p 384-391.

22. Horak I, Camicas J, Keirans E. 2002. The Argasidae, Ixodidae and Nutallie-llidae (Acari: Ixodida): a world list of valid tick names. Exp Appl Acarol 28: 27-54.

23. Huerto E, Dámaso B. 2015. Factores asociados a la infección por Ehrlichia canis en perros infestados con garrapatas en la ciudad de Huánuco, Perú. Rev Peru Med Exp Salud Publica 32: 756-760.

24. Ismail N, Bloch KC, McBride JW. 2010. Human ehrlichiosis and anaplasmosis. Clin Lab Med 30: 261-292. doi: 10.1016/j.cll.2009.10.004

25. Lindgren E, Tälleklint L, Polfeldt T. 2000. Impact of climatic change on the northern latitude limit and population density of the disease-transmitting European tick Ixodes ricinus. Environ Health Perspect 108: 119-123.

26. Martínez D, Oliver O. 2016. Alcalosis metabólica hipoclorémica o alcalosis de iones fuertes: una revisión. Rev Med Vet 32: 131-141. doi: 10.19052/mv.3862

27. Needham G, Teel P. 1991. Off host physiological ecology of ixodid ticks. Annu Rev Entomol 36: 659-681. doi: 10.1146/annurev.en.36.010191.003303

28. Peña I, Vidal F, Hernández A. 2016. Población de perros callejeros del municipio Camagüey, Cuba. Rev Inv Vet Perú 27: 840-844. doi: 10.15381/rivep.v27i4.12570

29. Pereira M, Labruna M, Szabó P, Klafke, G. 2008. Rhipicephalus (Boophilus) microplus: biologia, controle e resistência. São Paulo: MedVet Livros. $159 \mathrm{p}$.

30. Plumb D. 2010. Manual de farmacología veterinaria. $6^{a}$ ed. Buenos Aires: Inter-Médica. $820 \mathrm{p}$.

31. Tasayco R, Vásquez M, Pineda $C$, Chuquiyauri M. 2015. Estudio comparativo de dos tratamientos (tetraciclina clorhidrato y doxiciclina) contra la ehrlichiosis canina. Invest Valdizana 10(2): 55-60.

32. Wall R, Shearer D. 2001. Ticks (Acari). Ch 3. In: Veterinary ectoparasites: biology, pathology, and control. $2^{\text {nd }}$ ed. Oxford: Blackwell Science. p 55-82.

33. Woody B, Hoskins J. 1991. Ehrlichial diseases of dogs. Vet Clin North Am. Small Anim Pract 21: 75-98. doi: 10.1016/S0195-5616(91)50009-7 